\title{
Review
}

\section{Management of Visual Dysfunction in Patients with Parkinson's Disease}

\author{
Joseph Savitt ${ }^{\mathrm{a}, *}$ and Rachid Aouchiche ${ }^{\mathrm{b}, *}$ \\ ${ }^{a}$ Department of Neurology, University of Maryland School of Medicine, Baltimore, MD, USA \\ ${ }^{\mathrm{b}}$ Department of Ophthalmology and Visual Sciences, University of Maryland School of Medicine, \\ Neuro-Ophthalmology, Baltimore, MD, USA
}

Accepted 7 July 2020

\begin{abstract}
Parkinson's disease (PD) is a movement disorder with many symptoms responsive to treatment with dopamine agonists, anti-cholinergics and the dopamine precursor, levodopa. The cardinal features of PD include tremor, rigidity, bradykinesia, and postural instability. There also are non-motor features that include sleep disorders, cognitive and affective dysfunction, hyposmia, pain and dysautonomia (constipation, bloating, orthostasis, urinary symptoms, sexual dysfunction, dysphagia). Among these non-motor features are signs and symptoms of visual system impairment that range from subtle examination findings to those causing severe disability. In this review we describe common PD-related abnormalities in the visual system, how they present, and potential treatments.
\end{abstract}

Keywords: Parkinson's disease, vision, hallucination, diplopia, dry eye

\section{SCOPE OF VISUAL SYSTEM IMPAIRMENT IN PARKINSON'S DISEASE}

Pathology in Parkinson's disease (PD) involving the visual system occur at all levels of visual processing from the ocular environment where light is first detected to cortical areas that mediate interpretation of visual inputs. A recent survey found increased self-reporting of visual impairment in PD patients compared to controls (odds ratio 2.67) [1]. Another study of 848 PD patients and 250 age-matched con-

\footnotetext{
*Correspondence to: Joseph Savitt, MD, PhD, Department of Neurology, University of Maryland, School of Medicine, 110 South Paca Street, 3rd floor, Baltimore, MD 21201, USA. Tel.: +1 410328 7809; Fax: +1 410328 0167; E-mail: jsavitt@som.umaryland.edu and Rachid Aouchiche MD, FACS Assistant Professor, Department of Ophthalmology and Visual Sciences, University of Maryland School of Medicine, Neuro-Ophthalmology, Baltimore, MD 21201, USA. E-mail: raouchiche@som.umaryland.edu.
}

trols used a detailed 17 question instrument (Visual Impairment in Parkinson's Disease Questionnaire or VIPD-Q) and found that $82 \%$ of patients reported visual symptoms compared to $48 \%$ of controls. Symptoms included hallucinations, and other symptoms likely resulting from pathology in the eye, ocular surface, optic nerve and oculomotor function [2]. Visual symptoms interfered with activities of daily living in $68 \%$ of patients versus $35 \%$ of controls. In addition, people with PD were more likely to see an ophthalmologist, have more ophthalmologic complaints, use a visual aid and report visual aid inadequacy. PD patients had more driving difficulties and more frequent falls.

Overall visual function has been reported to be slower in PD as modeled in a picture identification task (MULES test - Mobile Universal Lexicon Evaluation System) and this correlated with disease severity [3]. In addition, PD patients made more naming errors and seemed to show a larger 
re-test improvement effect than controls, though a ceiling effect in controls could be responsible. Future studies should compare MULES to other visual assessments in PD and determine its clinical relevance.

\section{EYELID AND OCULAR ABNORMALITIES}

Abnormal visual function in PD may involve abnormal eyelid function (usually reduced blink rate, but rarely apraxia of eye opening or reflex blepharospasm), multiple ocular film abnormalities, corneal abnormalities, meibomian gland stenosis/blockage, decreased tear production and seborrheic blepharitis [4-6]).

Dry eye affects $53-87 \%$ of patients with PD and is associated with reduced blink rate as low as 1-2 per minute [6, 7]. Decreased blink rate results in increased tear evaporation by reducing the tear film lipid layer, particularly in the presence of associated blepharitis [8]. This in turn may result in increased ocular inflammation and worsening symptoms. Besides reduced blink rate, autonomic dysfunction affecting the lacrimal glands may result in ocular surface abnormalities [6]. Lid abnormalities, including reflex blepharospasm, can lead to added ocular discomfort, vision impairment and light sensitivity. In addition, the PD drug amantadine and other dopaminergic agents have been linked to reversible corneal edema with subsequent vision change $[9,10]$.

In our experience patients with ocular surface/eyelid abnormalities complain of dry eye, blurred vision, excessive tearing, foreign body sensation, glare, light sensitivity, problems keeping the eye open and general eye discomfort.

Traditional interventions (not PD-specific) to ease symptoms include treatment of blepharitis with lid hygiene, and dry eye with artificial tears, moisture chambers, nutritional supplements, scleral lenses and tear duct occlusion $[11,12]$ and interested readers are referred to an excellent review detailing diagnosis and treatment of dry eye in general, including level of evidence for particular treatments [13]. Blepharospasm responds to botulinum toxin injection of the eyelids [14].

Future research into dry eye treatment should include testing novel eyedrop formulations such as those containing blood and platelet-derived components and tear proteins (thymosin $\beta 4$ and lubricin) well as drugs to increase tear production (lactritin, diquafosol, rebamipide) and those to reduce inflam- mation [15]. There are procedures (meibomian gland probing, intense pulsed light, vector pulsed light therapy) and other physical methods using applied warmth and physical expression of meibum and devices (intranasal lacrimal gland stimulation, scleral lens) in development to protect the eye, treat blepharitis and to unblock meibomian gland ducts [15]. The effectiveness of these newer interventions is unclear in the general population as well as in those with PD thus requiring more study.

The potential severity of eyelid and ocular abnormalities in PD, their high incidence, the generally more advanced age of PD patients and the potential contributions of PD medications on pathology leads us to strongly encourage routine ophthalmological examination. Those having on-going symptoms are referred more urgently. Though no PD-specific guidelines exist, we recommend at least once a year ophthalmological follow up which is only a bit more aggressive than the once every 1-2 years recommended by the National Eye Institute for everyone over age 60 (http://www.nei.nih.gov).

\section{DIPLOPIA AND OCULOMOTOR ABNORMALITIES}

Ocular motility abnormalities in PD include saccadic intrusions, vestibulo-ocular reflex abnormalities, increased square-wave jerks associated with fixation, as well as other saccade and pursuit abnormalities [16-18]. Oculomotor abnormalities however are not as prominent as in other Parkinson's-related disorders, such as progressive supranuclear palsy [19] and their clinical relevance is unclear. It has been shown, however, that saccadic reading assessed by the King-Devick test in PD patients is $20 \%$ slower than controls [20] suggesting a potential role for abnormal extraocular movements in clinical impairment.

Diplopia is present in 10-30\% of PD patients [7] and occurs more frequently as PD progresses [21]. Convergence insufficiency $(\mathrm{CI})$ is prevalent in $\mathrm{PD}$ and often is associated with binocular horizontal diplopia at near. It is experienced during reading, and is exacerbated by dry eyes or ocular surface irritation, particularly with reduction in blink rate [22].

One should differentiate binocular diplopia due to $\mathrm{CI}$ or pre-existing strabismus from monocular diplopia generally resulting from refractive errors, corneal pathology, cataract formation or macular disorders that would have more specific ophthalmological treatments (e.g., cataract removal, refraction 
and spectacle correction, macular and corneal treatments). Selective or cortical diplopia (diplopia of single objects) is a rare phenomenon associated with visual hallucinations [23].

Management of CI by the orthoptist and the ophthalmologist includes single vision reading glasses, base-in prisms or monocular occlusion. We counsel against focal or progressive lenses as they tend to impede binocular vision and prevent the use of prisms in the lower portion of the lens. Patients may benefit from typoscopes, E-tablets and proper lighting to facilitate reading [24].

Intermittent diplopia at near related to CI may improve with prismatic correction and optimization of levodopa (especially if visual complaints are related to waning of dopaminergic medication effect, i.e., wearing off) [25]. In one study of PD patients, nearly $20 \%$ reported that levodopa treatment improved diplopia though this was not verified experimentally [26], while convergence did measurably improve in patients in the on-state (90 minutes after taking levodopa) versus prior to their routine levodopa dosing when the medication had started to wear off [27]. The authors suggest that medicationinduced fluctuations in convergence ability may make proper prism therapy difficult and this is important as prism placement is likely the most common intervention used in all adults for this condition [28].

\section{RETINAL AND OPTIC NERVE ABNORMALITIES}

Optical coherence tomography (OCT) has emerged as a tool in the assessment of retinal changes in PD, often with conflicting findings, reviewed in [29]. Despite the lack of reproducibility in retinal OCT studies, there are likely retina changes in PD with patterns of thinning possibly related to cellular or vascular changes [29]. Abnormal accumulation of retinal alpha-synuclein (whose abnormal accumulation in cells helps to pathologically define PD in the brain) is further evidence of retinal pathology in patients with PD [30, 31]. A recent meta-analysis of 36 studies determined that patients with PD have significantly thinner retinae compared to gender and age-matched controls [32]. This study also noted the similarity of inner retinal layer thinning seen in PD with those of other neurodegenerative disorders.
There is an increased incidence of glaucoma-like changes (optic nerve appearance, cup to disc ratio and glaucoma-like visual field changes) in PD, despite lower average intraocular pressures [4]. Hypotheses linking PD to a glaucoma-like condition have included the role of progressive dopamine depletion and alpha-synuclein induced axonal degeneration [33]. Clinical and pathology similarities and differences between PD-related optic nerve changes and what is commonly referred to as glaucoma require more investigation as does the role of standard glaucoma treatments in PD-related retinal disease.

Symptoms related to retinal pathology include reduced visual acuity, visual field defects, color vision impairment (though central mechanisms may be more important [34]), and reduced contrast sensitivity $[35,36]$.

Treating retinal and optic nerve pathology involves the optimization of levodopa therapy (found to improve contrast sensitivity and color vision [37, 38]) and ophthalmologic referral for glaucoma evaluation and acuity optimization. New drugs used to treat normal tension glaucoma through mechanisms that rescue nerve fibers may be most applicable to PD and such studies are on-going. For a summary of pathologies and treatments, see Table 1.

Finally, it is important to educate patients on the benefits of increased levels of lighting and to provide warnings about driving, especially in low contrast environments (fog or twilight) as contrast specificity may be a significant factor in driving fitness [39].

\section{HALLUCINATIONS AND VISUOPERCEPTUAL IMPAIRMENT}

Visual hallucinations are reported by about $30 \%$ of PD patients, with a lifetime prevalence of up to $60-80 \%$ [40, 41]. Often hallucinations are accompanied or preceded by illusions (visual misinterpretations), or a false sense of presence or something moving in the environment. Hallucinations occur with or without insight into their false nature, the latter being more problematic. PD-related hallucinations negatively impact quality of life, ability to live independently and on mortality [11].

Hallucinations and visual misperceptions can be caused by pathology all along the visual pathway from impaired visual sensory input due to ocular changes back through pathological changes in the cortex [42]. Genetic polymorphisms, disease 
Table 1

Ophthalmic pathology and management in Parkinson's disease. Treatment recommendations are often not PD-specific and are taken from general ophthalmologic practice, literature review and author's experience. See text for details

Eyelid/Ocular surface

- Decreased blink rate

- Dry eye syndrome

- Blepharitis

- Meibomian gland dysfunction

- Blepharospasm

Treatment

- Preservative-free artificial tears [7]

- Tear duct occlusion [7]

- Lid hygiene [7]

- Botox for blepharospasm [14]

- Increase in dopaminergic agents

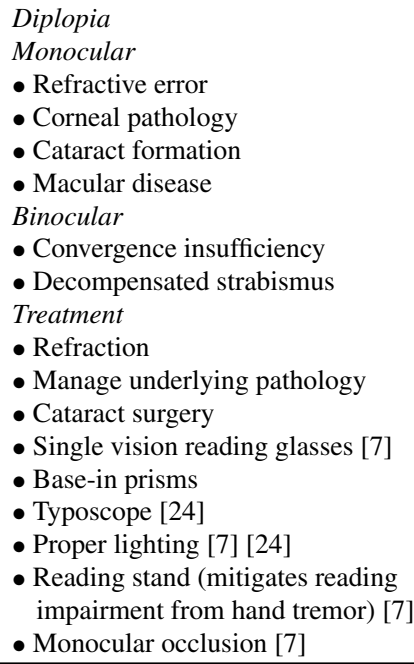

Monocular

- Refractive error

- Corneal pathology

- Cataract formation

- Macular disease

Binocular

- Convergence insufficiency

- Decompensated strabismus

Treatment

- Refraction

- Manage underlying pathology

- Cataract surgery

- Single vision reading glasses [7]

- Base-in prisms

- Typoscope [24]

- Proper lighting [7] [24]

- Reading stand (mitigates reading

impairment from hand tremor) [7]

- Monocular occlusion [7]

\author{
Visual loss and visuospatial impairment \\ Retina/optic nerve \\ - Decreased acuity and color vision \\ - Reduced contrast sensitivity \\ - Visual field defects \\ Cortical \\ - Visual hallucinations \\ Treatment \\ - Visual rehabilitation [24] \\ - Occupational therapy [24] \\ - Dopaminergic therapy [37] \\ - See Figure 1 for hallucination algorithm
}

If hallucinations persist, a reduction of levodopa

severity, age, sleep disturbances, dementia and certain medications use contribute to hallucination risk [11, 41, 43-45]. Impaired vision due to any cause increases the risk of visual hallucinations (Charles Bonnet syndrome) and causes such as cataracts and macular degeneration should be identified and treated.

The sudden onset or worsening of hallucinations can result from changes in medications or from an infectious/metabolic abnormality. In our experience, dehydration, and urinary tract infections as well as the initiation of pain or urinary (anti-cholinergic) medication are frequent, reversible causes.

Treatment of hallucinations may best begin prior to their development by preparing the patient and caregivers for their emergence. This helps to normalize this symptom and hopefully reduce the stigma and potential distress of their presence. We encourage patients to notify their physician of the emergence of hallucinations and to discuss whether they are causing enough distress to require treatment.

Treating problematic hallucinations involves tapering back contributory medications when possible and this includes every PD medication to varying degrees. The recent publication of guidelines resulting from a workshop on visual hallucinations in a variety of disorders, citing guidelines from the National Institute for Health and Care Excellence from 2017, were incorporated into the strategy for addressing PD-related hallucinations found in Fig. 1 [11]. Levodopa monotherapy for PD symptoms should be the eventual goal. dose including the use of lower dose strengths, perhaps given more often (to reduce levodopa peak plasma levels) should be attempted. In carefully screened patients who require anticholinergics or levodopa to control symptoms, the dose requirement can be reduced using subthalamic nucleus (STN) deep brain stimulation to mitigate side effects, including hallucinations $[46,47]$.

If medication adjustment does not improve hallucinations, we consider adding medications to directly target the problem including cholinesterase inhibitors and antipsychotics.

Several studies offer evidence of the potential benefit of cholinesterase inhibitors in PD-related hallucinations, especially in the presence of concomitant dementia [48, 49]. Potential side effects including GI upset/bleeding, sialorrhea, increased tremor, syncope, seizures, and arrhythmia, among others, need to be considered.

Antipsychotics can improve PD hallucinations. The most often employed are quetiapine, clozapine and pimavanserin as others often worsen motor function at therapeutic doses. Other side effects including QTc prolongation and increased mortality must be considered [50, 51]. The use of quetiapine for PD-related hallucinations is common, though clear evidence of benefit is generally lacking [52]. Despite this, the perception by many is that quetiapine is generally well-tolerated when kept at doses at or below $100-150 \mathrm{mg}$ daily, is beneficial for overnight sleep when dosed at bedtime and is more 


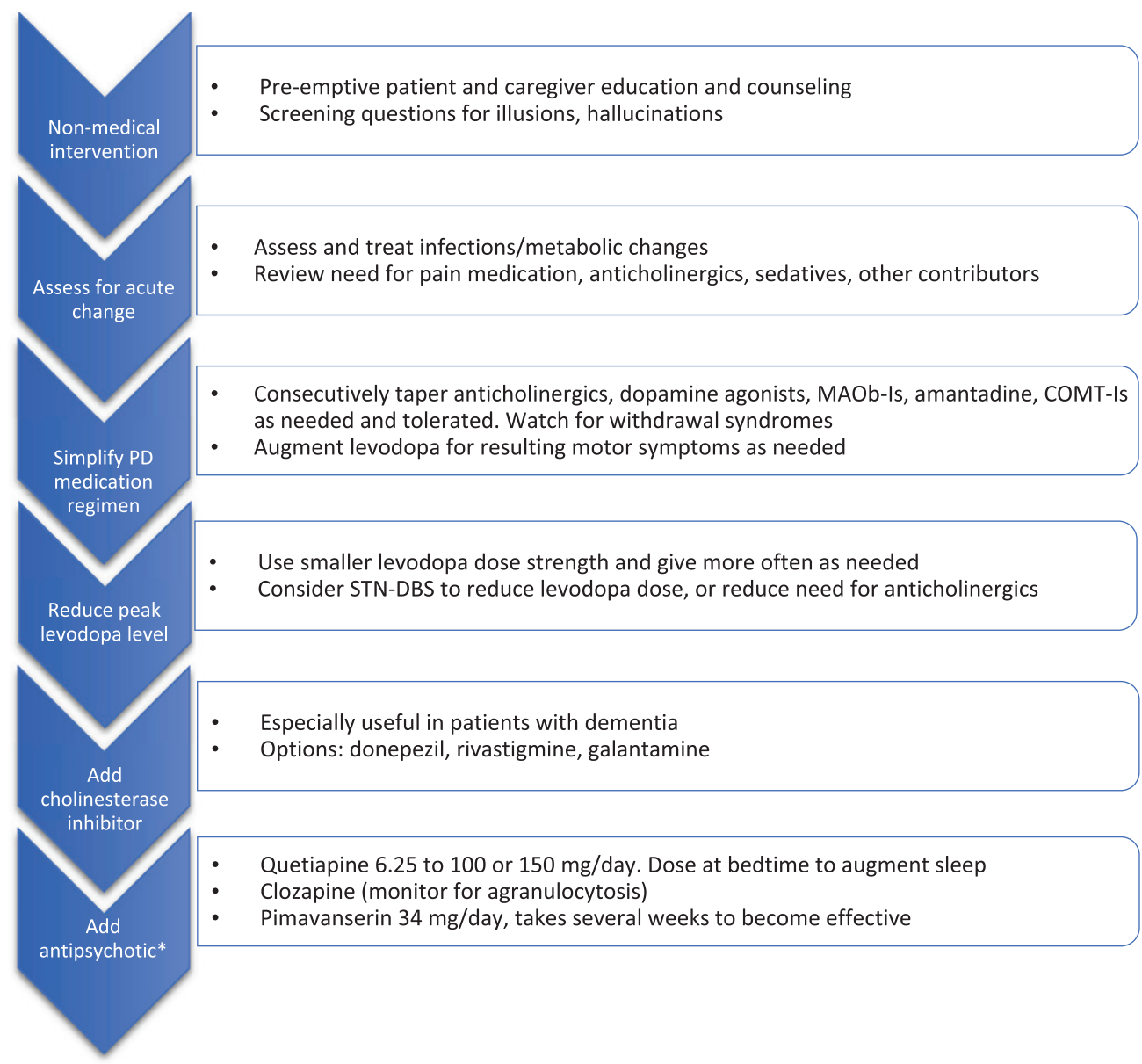

\section{Adequate Hallucination}

Control

Fig. 1. Addressing hallucinations in Parkinson's disease. Flow chart for stepwise treatment of hallucinations in PD. See text for details and additional warnings. *Use of antipsychotics in patients suffering from dementia must proceed with caution given the black box warning for increased mortality, also QTc should be monitored. MAOb-I, monoamine oxidase B inhibitor; STN-DBS, subthalamic nucleus-deep brain stimulation; COMT-I, catechol- $O$-methyltransferase inhibitor.

convenient than using Clozapine and pimavanserin (see below).

Pimavanserin is the only medication approved for the treatment of PD psychosis and likely works via serotonin 2a (5-HT2a) receptor inverse agonism. A recent meta-analysis of four relevant studies demonstrated efficacy on a variety of measures and a lack of significant motor side effects [53]. It does require several weeks to take effect and is more expensive than quetiapine.

Clozapine reduces hallucinations in PD patients at daily doses ranging from $12.5 \mathrm{mg}$ to $50 \mathrm{mg}$ without significant effect on motor function [54]. Despite proven efficacy and introduction decades ago, widespread use is limited by concerns regarding granulocytopenia and the frequent laboratory monitoring required for its use.

Importantly, control of hallucinations with additional agents such as clozapine allows levodopa doses to be increased and better optimized, and other antiParkinson medications to be used with a reduced risk of hallucinations returning [55].

Addressing visual hallucinations continues to be an unmet need in PD therapy. The presence of 
hallucinations continues to limit medication options and may continue to be present despite best therapy. Future areas of potential research include options to improve visual acuity through treatment of concurrent condition (such as experimental treatment of macular degeneration) or therapies that may improve PD-related retinal degeneration. A clue to potential therapies comes from case reports addressing hallucinations in Charles Bonnet syndrome where a variety of available medications including gabapentin, serotonergic agents as well as transcranial magnetic stimulation may offer promise (reviewed in [11]). There are on-going trials assessing the potential benefit of ondansetron and novel antipsychotics such as SEP363856 (affecting serotinergic and TAAR1 receptors). Nelotanserin, whose mechanism parallels that of pimvansenin is in clinical trials. Saracatinib, a kinase inhibitor postulated to block 5HT-2a receptor effects via inhibition of the downstream Src kinase, is also under study.

\section{CONCLUSIONS AND FUTURE DIRECTIONS}

There is increased awareness of visual dysfunction as a "non-motor" feature of PD. The availability of reporting tools such as the VIPD-Q will lead to increased awareness of visual symptoms in patients with PD and help standardize research in the field. Advances and optimization of OCT will demonstrate the nature of retinal changes that may help in diagnosis, prognosis and treatment not only for visual symptoms, but also for PD in general, as several studies have showed an association between retinal thinning and disease progression and severity. There is a current study examining the association of OCT changes with PD progression and to identify possible PD patients from high risk populations prior to diagnosis. Interventions to treat dry eye and glaucoma may be generalizable to patients with PD and further research is needed to examine this. From a practical standpoint, early recognition and management of visual disorders in PD is important to prevent further deterioration and unnecessary disability. Visual changes impact driving and falling in PD and proper treatment and counseling can reduce these hazards. Physicians are encouraged to ask patients about various symptoms of ocular involvement and refer to ophthalmologists or neuro-ophthalmologists.

\section{CONFLICT OF INTEREST}

The authors have no conflict of interest to report.

\section{REFERENCES}

[1] Hamedani AG, Willis AW (2020) Self-reported visual dysfunction in Parkinson disease: The Survey of Health, Ageing and Retirement in Europe. Eur J Neurol 27, 484-489.

[2] Borm C, Visser F, Werkmann M, de Graaf D, Putz D, Seppi K, Poewe W, Vlaar AMM, Hoyng C, Bloem BR, Theelen T, de Vries NM (2020) Seeing ophthalmologic problems in Parkinson disease: Results of a visual impairment questionnaire. Neurology 94, e1539-e1547.

[3] Conway J, Ilardi M, Gonzalez C, Dahan N, Fallon S, Moehringer N, Hasanaj L, Joseph B, Serrano L, Rizzo JR, Rucker JC, Feigin A, Frucht S, Galetta SL, Balcer LJ (2020) Rapid picture naming in Parkinson's disease using the Mobile Universal Lexicon Evaluation System (MULES). J Neurol Sci 410, 116680.

[4] Nowacka B, Lubinski W, Honczarenko K, Potemkowski A, Safranow K (2014) Ophthalmological features of Parkinson disease. Med Sci Monit 20, 2243-2249.

[5] Kimura N, Watanabe A, Suzuki K, Toyoda H, Hakamata N, Fukuoka H, Washimi Y, Arahata Y, Takeda A, Kondo M, Mizuno T, Kinoshita S (2017) Measurement of spontaneous blinks in patients with Parkinson's disease using a new high-speed blink analysis system. J Neurol Sci $\mathbf{3 8 0}$, 200-204.

[6] Tamer C, Melek IM, Duman T, Oksuz H (2005) Tear film tests in Parkinson's disease patients. Ophthalmology 112, 1795.

[7] Biousse V, Skibell BC, Watts RL, Loupe DN, Drews-Botsch C, Newman NJ (2004) Ophthalmologic features of Parkinson's disease. Neurology 62, 177-180.

[8] Bowen RC, Koeppel JN, Christensen CD, Snow KB, Ma J, Katz BJ, Krauss HR, Landau K, Warner JEA, Crum AV, Straumann D, Digre KB (2018) The most common causes of eye pain at 2 tertiary ophthalmology and neurology clinics. J Neuroophthalmol 38, 320-327.

[9] Daggumilli S, Vanathi M, Ganger A, Goyal V, Tandon R (2019) Corneal evaluation in patients with parkinsonism on long-term amantadine therapy. Cornea 38, 1131-1136.

[10] Mancera N, Wadia HP (2019) Corneal edema associated with systemic dopaminergic agents. Cornea 38, 1040-1042.

[11] O'Brien J, Taylor JP, Ballard C, Barker RA, Bradley C, Burns A, Collerton D, Dave S, Dudley R, Francis P, Gibbons A, Harris K, Lawrence V, Leroi I, McKeith I, Michaelides M, Naik C, O'Callaghan C, Olsen K, Onofrj M, Pinto R, Russell G, Swann P, Thomas A, Urwyler P, Weil RS, Ffytche D (2020) Visual hallucinations in neurological and ophthalmological disease: Pathophysiology and management. J Neurol Neurosurg Psychiatry 91, 512-519.

[12] Savitt J, Mathews M (2018) Treatment of visual disorders in Parkinson disease. Curr Treat Options Neurol 20, 30.

[13] Milner MS, Beckman KA, Luchs JI, Allen QB, Awdeh RM, Berdahl J, Boland TS, Buznego C, Gira JP, Goldberg DF, Goldman D, Goyal RK, Jackson MA, Katz J, Kim T, Majmudar PA, Malhotra RP, McDonald MB, Rajpal RK, Raviv T, Rowen S, Shamie N, Solomon JD, Stonecipher K, Tauber S, Trattler W, Walter KA, Waring GOt, Weinstock RJ, Wiley WF, Yeu E (2017) Dysfunctional tear syndrome: Dry eye 
disease and associated tear film disorders - new strategies for diagnosis and treatment. Curr Opin Ophthalmol 27 Suppl 1, 3-47.

[14] Green KE, Rastall D, Eggenberger E (2017) Treatment of blepharospasm/hemifacial spasm. Curr Treat Options Neurol 19, 41 .

[15] O'Neil EC, Henderson M, Massaro-Giordano M, Bunya VY (2019) Advances in dry eye disease treatment. Curr Opin Ophthalmol 30, 166-178.

[16] Leigh RJ, Zee DS (2015) The neurology of eye movements, Oxford University Press, Oxford; New York.

[17] Terao Y, Fukuda H, Ugawa Y, Hikosaka O (2013) New perspectives on the pathophysiology of Parkinson's disease as assessed by saccade performance: A clinical review. Clin Neurophysiol 124, 1491-1506.

[18] Lv W, Guan Q, Hu X, Chen J, Jiang H, Zhang L, Fan W (2017) Vestibulo-ocular reflex abnormality in Parkinson's disease detected by video head impulse test. Neurosci Lett 657, 211-214.

[19] Jung I, Kim JS (2019) Abnormal eye movements in parkinsonism and movement disorders. J Mov Disord 12, 1-13.

[20] Jehangir N, Yu CY, Song J, Shariati MA, Binder S, Beyer J, Santini V, Poston K, Liao YJ (2018) Slower saccadic reading in Parkinson's disease. PLoS One 13, e0191005.

[21] Archibald NK, Clarke MP, Mosimann UP, Burn DJ (2011) Visual symptoms in Parkinson's disease and Parkinson's disease dementia. Mov Disord 26, 2387-2395.

[22] Ekker MS, Janssen S, Seppi K, Poewe W, de Vries NM, Theelen T, Nonnekes J, Bloem BR (2017) Ocular and visual disorders in Parkinson's disease: Common but frequently overlooked. Parkinsonism Relat Disord 40, 1-10.

[23] Nebe A, Ebersbach G (2007) Selective diplopia in Parkinson's disease: A special subtype of visual hallucination? Mov Disord 22, 1175-1178.

[24] Fletcher DC (1999) Low vision rehabilitation: Caring for the whole person, American Academy of Ophthalmology, San Franciso.

[25] Racette BA, Gokden MS, Tychsen LS, Perlmutter JS (1999) Convergence insufficiency in idiopathic Parkinson's disease responsive to levodopa. Strabismus 7, 169-174.

[26] Schindlbeck KA, Schonfeld S, Naumann W, Friedrich DJ, Maier A, Rewitzer C, Klostermann F, Marzinzik F (2017) Characterization of diplopia in non-demented patients with Parkinson's disease. Parkinsonism Relat Disord 45, $1-6$.

[27] Almer Z, Klein KS, Marsh L, Gerstenhaber M, Repka MX (2012) Ocular motor and sensory function in Parkinson's disease. Ophthalmology 119, 178-182.

[28] Ghadban R, Martinez JM, Diehl NN, Mohney BG (2015) The incidence and clinical characteristics of adult-onset convergence insufficiency. Ophthalmology 122, 10561059.

[29] Mailankody P, Lenka A, Pal PK (2019) The role of optical coherence tomography in parkinsonism: A critical review. J Neurol Sci 403, 67-74.

[30] Chorostecki J, Seraji-Bozorgzad N, Shah A, Bao F, Bao G, George E, Gorden V, Caon C, Frohman E, Bhatti MT, Khan O (2015) Characterization of retinal architecture in Parkinson's disease. J Neurol Sci 355, 44-48.

[31] Bodis-Wollner I, Kozlowski PB, Glazman S, Miri S (2014) alpha-synuclein in the inner retina in parkinson disease. Ann Neurol 75, 964-966.

[32] Chrysou A, Jansonius NM, van Laar T (2019) Retinal layers in Parkinson's disease: A meta-analysis of spectral-domain optical coherence tomography studies. Parkinsonism Relat Disord 64, 40-49.

[33] Nucci C, Martucci A, Cesareo M, Garaci F, Morrone LA, Russo R, Corasaniti MT, Bagetta G, Mancino R (2015) Links among glaucoma, neurodegenerative, and vascular diseases of the central nervous system. Prog Brain Res 221, 49-65.

[34] Bohnen NI, Haugen J, Ridder A, Kotagal V, Albin RL, Frey KA, Muller M (2017) Color discrimination errors associate with axial motor impairments in Parkinson's disease. Mov Disord Clin Pract 4, 864-869.

[35] Satue M, Rodrigo MJ, Obis J, Vilades E, Gracia H, Otin S, Fuertes MI, Alarcia R, Crespo JA, Polo V, Larrosa JM, Pablo LE, Garcia-Martin E (2017) Evaluation of progressive visual dysfunction and retinal degeneration in patients with Parkinson's disease. Invest Ophthalmol Vis Sci 58, 11511157.

[36] Polo V, Satue M, Rodrigo MJ, Otin S, Alarcia R, Bambo MP, Fuertes MI, Larrosa JM, Pablo LE, Garcia-Martin E (2016) Visual dysfunction and its correlation with retinal changes in patients with Parkinson's disease: An observational crosssectional study. BMJ Open 6, e009658.

[37] Buttner T, Kuhn W, Patzold T, Przuntek H (1994) L-Dopa improves colour vision in Parkinson's disease. J Neural Transm Park Dis Dement Sect 7, 13-19.

[38] Hutton JT, Morris JL, Elias JW (1993) Levodopa improves spatial contrast sensitivity in Parkinson's disease. Arch Neurol 50, 721-724.

[39] Alvarez L, Classen S (2018) Driving with Parkinson's disease: Cut points for clinical predictors of on-road outcomes: La conduite automobile et la maladie de Parkinson: Points de decoupage pour les predicteurs clinique des resultats des epreuves sur route. Can J Occup Ther 85, 232-241.

[40] Gibson G, Mottram PG, Burn DJ, Hindle JV, Landau S, Samuel M, Hurt CS, Brown RG, KC MW (2013) Frequency, prevalence, incidence and risk factors associated with visual hallucinations in a sample of patients with Parkinson's disease: A longitudinal 4-year study. Int J Geriatr Psychiatry 28, 626-631.

[41] Diederich NJ, Fenelon G, Stebbins G, Goetz CG (2009) Hallucinations in Parkinson disease. Nat Rev Neurol 5, 331342.

[42] Kurita A, Koshikawa H, Akiba T, Seki K, Ishikawa H, Suzuki M (2019) Visual hallucinations and impaired conscious visual perception in Parkinson disease. J Geriatr Psychiatry Neurol. doi: 10.1177/0891988719892318

[43] Damasceno Dos Santos EU, Duarte EBC, Miranda LMR, Asano AGC, Asano NMJ, Maia MMD, de Souza PRE (2019) Pharmacogenetic profile and the occurrence of visual hallucinations in patients with sporadic Parkinson's disease. J Clin Pharmacol 59, 1006-1013.

[44] Russo M, Carrarini C, Dono F, Rispoli MG, Di Pietro M, Di Stefano V, Ferri L, Bonanni L, Sensi SL, Onofrj M (2019) The pharmacology of visual hallucinations in synucleinopathies. Front Pharmacol 10, 1379.

[45] Papapetropoulos S, Argyriou AA, Ellul J (2005) Factors associated with drug-induced visual hallucinations in Parkinson's disease. J Neurol 252, 1223-1228.

[46] Yoshida F, Miyagi Y, Kishimoto J, Morioka T, Murakami N, Hashiguchi K, Samura K, Sakae N, Yamasaki R, Kawaguchi M, Sasaki T (2009) Subthalamic nucleus stimulation does not cause deterioration of preexisting hallucinations in Parkinson's disease patients. Stereotact Funct Neurosurg 87, 45-49. 
[47] Umemura A, Oka Y, Okita K, Matsukawa N, Yamada K (2011) Subthalamic nucleus stimulation for Parkinson disease with severe medication-induced hallucinations or delusions. J Neurosurg 114, 1701-1705.

[48] Kurita A, Ochiai Y, Kono Y, Suzuki M, Inoue K (2003) The beneficial effect of donepezil on visual hallucinations in three patients with Parkinson's disease. J Geriatr Psychiatry Neurol 16, 184-188.

[49] Sobow T (2007) Parkinson's disease-related visual hallucinations unresponsive to atypical antipsychotics treated with cholinesterase inhibitors: A case series. Neurol Neurochir Pol 41, 276-279.

[50] Weintraub D, Chiang C, Kim HM, Wilkinson J, Marras C, Stanislawski B, Mamikonyan E, Kales HC (2017) Antipsychotic use and physical morbidity in Parkinson disease. Am J Geriatr Psychiatry 25, 697-705.

[51] Weintraub D, Chiang C, Kim HM, Wilkinson J, Marras C, Stanislawski B, Mamikonyan E, Kales HC (2016) Association of antipsychotic use with mortality risk in patients with Parkinson disease. JAMA Neurol 73, 535-541.
[52] Chen JJ, Hua H, Massihi L, Portillo I, Alipour A, Ondo W, Dashtipour K (2019) Systematic literature review of quetiapine for the treatment of psychosis in patients with parkinsonism. J Neuropsychiatry Clin Neurosci 31, 188195.

[53] Zhang H, Wang L, Fan Y, Yang L, Wen X, Liu Y, Liu Z (2019) Atypical antipsychotics for Parkinson's disease psychosis: A systematic review and meta-analysis. $\mathrm{Neu}$ ropsychiatr Dis Treat 15, 2137-2149.

[54] Pollak P, Tison F, Rascol O, Destee A, Pere JJ, Senard JM, Durif F, Bourdeix I (2004) Clozapine in drug induced psychosis in Parkinson's disease: A randomised, placebo controlled study with open follow up. J Neurol Neurosurg Psychiatry 75, 689-695.

[55] Factor SA, Brown D (1992) Clozapine prevents recurrence of psychosis in Parkinson's disease. Mov Disord 7, 125-131. 